\title{
Role of the Auxiliary Ligand in the
}

\section{Spontaneous Resolution of Enantiomers in Three-}

\section{Dimensional Coordination Polymers}

Hui-Jun Chen ${ }^{1}$, Lu Xu ${ }^{1}$, Man-Ting Chen ${ }^{1}$, Li-Rong Lin ${ }^{1}$, Gui-Lin Zhuang ${ }^{2 *}$, La-Sheng Long ${ }^{1 *}$, and Lan-Sun Zheng ${ }^{l}$

${ }^{1}$ Collaborative Innovation Center of Chemistry for Energy Materials, State Key Laboratory of Physical Chemistry of Solid Surfaces and Department of Chemistry, College of Chemistry and Chemical Engineering, Xiamen University, Xiamen, 361005, China.

${ }^{2}$ College of Chemical Engineering, Zhejiang University of Technology, Hangzhou, 310032, P. R. China.

*Correspondence and requests for materials should be addressed to L.-S. Long and G.-L. Zhuang (E-mail: 1slong@xmu.edu.cn, glzhuang@zjut.edu.cn). 


\section{Syntheses of samples.}

Synthesis of $\left[\mathbf{Z n}_{\mathbf{2}}(\mathbf{B D C})(\mathbf{l a c})(\mathbf{D M F})\right] \cdot$ Guest $($ Guest $=1.5 \mathrm{DMF}+i-\mathrm{PrOH})(\mathbf{2})$

A mixture of $\mathrm{Zn}\left(\mathrm{NO}_{3}\right)_{2} \cdot 6 \mathrm{H}_{2} \mathrm{O}(0.5 \mathrm{mmol})$, benzene dicarboxylic acid $\left(\mathrm{H}_{2} \mathrm{BDC}, 0.33 \mathrm{mmol}\right)$ and racemic lactic acid ( $\mathrm{rac}-\mathrm{H}_{2}$ lac, 4 drops) was added to the mixed solution of $\mathrm{N}$,Ndimethylformamide (DMF, $5 \mathrm{~mL})$ and isopropanol $(i$-PrOH, $5 \mathrm{~mL})$. The resulting clear solution was sealed in a $23 \mathrm{~mL}$ Teflon-lined stainless steel container after stirring for a period of time. The container was heated to $140{ }^{\circ} \mathrm{C}$ and maintained at this temperature for 3 days and then cooled to $30{ }^{\circ} \mathrm{C}$ at a rate of $2{ }^{\circ} \mathrm{C} / \mathrm{h}$. Colorless block-shaped crystals of $\mathbf{2 D}$ and $\mathbf{2 L}$ (yield $61 \%$ based on zinc) were collected after washing with DMF. Anal. Calcd. for $2(\%)$ : C, 41.26\%; H, 5.40\%; N, 5.60\%. Found: C, 41.81\%; H, 5.30\%; N, 5.18\%.

Synthesis of $\left[\mathbf{C o}_{2}(\mathbf{B D C})(\mathbf{l a c})(\mathbf{D M F})\right] \cdot$ Guest $\left(\mathrm{Guest}=\mathrm{DMF}+2 \mathrm{H}_{2} \mathrm{O}\right)(\mathbf{3})$

Complex 3 was prepared in the similar way as described for 2 , except that $\mathrm{CoCl}_{2} \cdot 6 \mathrm{H}_{2} \mathrm{O}$ was used to replace $\mathrm{Zn}\left(\mathrm{NO}_{3}\right)_{2} \cdot 6 \mathrm{H}_{2} \mathrm{O}$. Violet block-shaped crystals of 3D and 3L (yield 56\% based on cobalt) were collected after washing with DMF. Anal. Calcd. for 3 (\%): C, 36.97\%; H, 4.75\%; N, 5.07\%. Found: C, 36.45\%; H, 4.39\%; N, 4.95\%.

Synthesis of $\left\{\left[\mathbf{F e}_{\mathbf{4}}(\mathbf{B D C})_{\mathbf{3}}(\mathbf{l a c})_{\mathbf{2}}(\mathbf{D M F})_{\mathbf{2}}\right]\left(\mathbf{C O}_{3}\right)\right\} \cdot$ Guest $\left(\right.$ Guest $\left.=\mathrm{DMF}+2 \mathrm{H}_{2} \mathrm{O}\right)(\mathbf{4})$

Complex 4 was prepared in the similar way as described for 2, except that $\mathrm{FeCl}_{3} \cdot 6 \mathrm{H}_{2} \mathrm{O}$ was used to replace $\mathrm{Zn}\left(\mathrm{NO}_{3}\right)_{2} \cdot 6 \mathrm{H}_{2} \mathrm{O}$. Brown block-shaped crystals of $\mathbf{4 D}$ and $\mathbf{4 L}$ (yield $68 \%$ based on $\mathrm{H}_{2} \mathrm{BDC}$ ) were collected after washing with DMF. Anal. Calcd. for 4 (\%): C, 39.80\%; H, 3.76\%; N, 3.48\%. Found: C, 40.22\%; H, 4.06\%; N, 3.33\%. 
Synthesis of $\left\{\mathbf{Z n}_{\mathbf{1 1}}(\mathbf{B P D C})_{\mathbf{6}}(\mathbf{l a c})_{6}\left[\mathbf{N H}_{\mathbf{2}}\left(\mathbf{C H}_{\mathbf{3}}\right)_{2}\right]_{2}\right\} \cdot$ Guest $\left(\right.$ Guest $\left.=6 \mathrm{DMF}+18 \mathrm{H}_{2} \mathrm{O}\right)(\mathbf{5})$

A mixture of $\mathrm{Zn}\left(\mathrm{NO}_{3}\right)_{2} \cdot 6 \mathrm{H}_{2} \mathrm{O}(0.5 \mathrm{mmol}), 3,3^{\prime}$-biphenyldicarboxylic acid $\left(\mathrm{H}_{2} \mathrm{BPDC}, 0.125 \mathrm{mmol}\right)$ and racemic lactic acid ( $\mathrm{rac}-\mathrm{H}_{2} \mathrm{lac}, 4$ drops) was added to the mixed solution of $\mathrm{N}, \mathrm{N}-$ dimethylformamide (DMF, $5 \mathrm{~mL}$ ) and ethanol (EtOH, $5 \mathrm{~mL}$ ). The resulting solution solution was then sealed in a $23 \mathrm{~mL}$ Teflon-lined stainless steel container after stirring for a period of time. The container was heated to $140{ }^{\circ} \mathrm{C}$ and maintained at this temperature for 4 days and then cooled to $30{ }^{\circ} \mathrm{C}$ at a rate of $2{ }^{\circ} \mathrm{C} / \mathrm{h}$. Colorless block-shaped crystals of $\mathbf{5 D}$ and $\mathbf{5 L}$ (yield $43 \%$ based on $\mathrm{H}_{2} \mathrm{BPDC}$ ) were collected after washing with EtOH. Anal. Calcd. for 5 (\%): C, 42.02\%; H, 4.72\%; N, 3.16\%. Found: C, 41.92\%; H, 4.86\%; N, 3.17\%.

\section{X-ray Crystallography}

Structural data of 3L, 4D, 4L, 5D, 5L were recorded by a XtaLAB Synergy four-circle diffractometer with monochromatic $\mathrm{Cu} \mathrm{K} \alpha$ radiation $(\lambda=1.54184 \AA)$, and the structural data of 2D and 2L were measured through an Agilent technologies Super Nova Micro Focus single Crystal Diffractometer with Mo-K $\alpha$ radiation $(\lambda=0.71013 \AA)$, and the structural data of 3D was collected by an Agilent technologies Super Nova Micro Focus single Crystal Diffractometer with $\mathrm{Cu}-\mathrm{K} \alpha$ radiation $(\lambda=1.54184 \AA)$. Absorption corrections were completed by using the multi-scan program. The above structures were solved by direct methods (ShelXT), ${ }^{1}$ and all the non-hydrogen atoms were refined aniostropically by full-matrix least-squares method on $F^{2}$ using ShelXL within Olex2. ${ }^{2-3}$ Except for 5D and $\mathbf{5 L}$, guest molecules were removed through SQUEEZE in structure refinement due to disorder. ${ }^{4}$ The X-ray crystallographic coordinates for structures reported in this article have been deposited at the Cambridge Crystallographic Data Center (CCDC). CCDC 
2044330-2044336, 2044660 and 2044619 contain the supplementary crystallographic data for 2D,

3D, 3L, 4D, 4L, 5D and 5L. The data can be obtained free of charge from the Cambridge Crystallographic Data Centre via www.ccdc.cam.ac.uk/data_request/cif.

\section{Computational detail}

Density functional theory (DFT) calculations were carried out by using Vienna ab-initio Simulation Package (VASP). ${ }^{5}$ The exchange-correlation term in the Kohn-shame equation was described with the generalized gradient approximation (GGA) and the Perdew-Burke-Ernzerhof (PBE). ${ }^{6}$ Projector-augmented wave (PAW) method of Blöchl, ${ }^{7-8}$ featuring the accuracy of augmented plane-wave methods as well as the efficiency of the pseudopotential approach, was used to deal with the interactions between ion and electrons. $3 \mathrm{~d}$ and $4 \mathrm{~s}$ electrons of Fe and $\mathrm{Zn}, 2 s$ and $2 p$ electrons of $\mathrm{C}, \mathrm{N}$ and $\mathrm{O}$, and $1 \mathrm{~s}$ electrons of $\mathrm{H}$ were explicitly treated as valence electrons. Van der Waals interactions were treated by using DFT-D3 method with Becke-Jonson damping. ${ }^{9}$ The cutoff energy for the plane wave basis sets was $450 \mathrm{eV}$. The convergence threshold of atoms position and cell parameters were set to be $10^{-5} \mathrm{eV}$ in energy and $10^{-2} \mathrm{eV} / \AA$ in force. The Brillouin zone was sampled by k-points mesh of $2 \times 2 \times 1$, which had be validated by convergence test. After geometrical optimization, single-point energy $\left(\mathbf{E}_{\mathbf{D F T}}\right)$ were obtained by using HSE-06 hybrid functional. ${ }^{10-12}$ Based on the relaxed structure, thermodynamic data except $\mathbf{E}_{\text {DFT }}$ were obtained by frequency analysis by using of PBE-D3 methods. Post-treatment for thermodynamic properties were conducted by VASPKIT program. ${ }^{13}$

$$
\begin{aligned}
& \Delta_{r} H=\Delta_{f} H(\text { product })-\left[\Delta_{f} H(\text { metal salt })+\Delta_{f} H(\text { ligand })\right] \\
& \Delta_{r} S=S(\text { product })-[S(\text { metal salt })+S(\text { ligand })]
\end{aligned}
$$




$$
\begin{aligned}
& \Delta\left(\Delta_{r} H\right)=\Delta_{r} H_{2}-\Delta_{r} H_{l}=\Delta_{f} H_{2}(\text { product })-\Delta_{f} H_{l}(\text { product }) \\
& \Delta\left(\Delta_{r} S^{*} T\right)=\left(\Delta_{r} S_{2}-\Delta_{r} S_{l}\right) T=S_{2}(\text { product }) T-S_{l}(\text { product }) T \\
& \left.\Delta\left(\Delta_{r} G\right)=\Delta_{r} G_{2}-\Delta_{r} G_{l}=\Delta\left(\Delta_{r} H\right)-\Delta_{(} \Delta_{r} S^{*} T\right)
\end{aligned}
$$

Owing to the same metal salt, together with equivalent electronic property of three ligands $\left(\mathrm{L}_{\mathrm{R}}, \mathrm{L}_{\mathrm{S}}\right.$ and L), the enthalpy change $\Delta_{\mathrm{r}} \mathbf{H}$ and the entropy change $\Delta_{\mathbf{r}} \mathbf{S}$ can be obtained as shown in eq(1)-

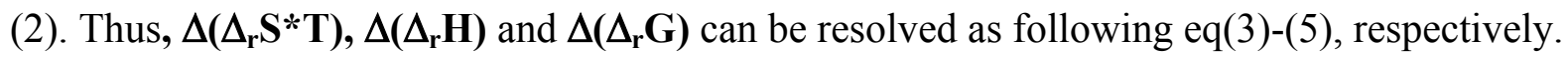

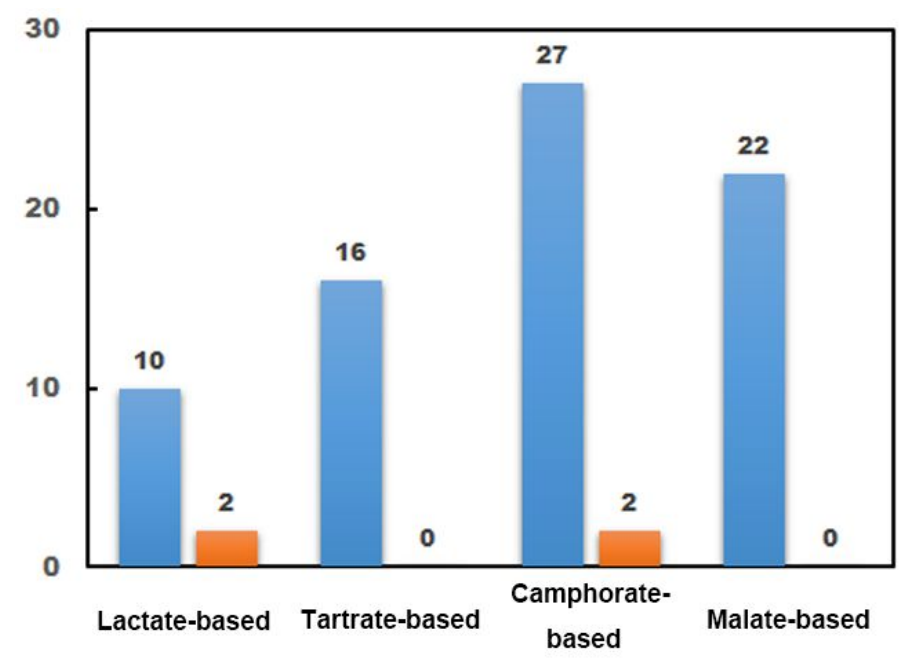

Figure S1. The percentages of spontaneous resolution in lactate-based, tartrate-based, camphoratebased and malate-based 3D CPs based on mixed ligand strategy. (The blue columns represents the total cases of $3 \mathrm{D}$ CPs prepared by racemic ligand, and the orange columns represents the spontaneous resolution cases among these 3D CPs. Data sources are from the Cambridge Crystallographic Data Center. ${ }^{14}$ ) 
a
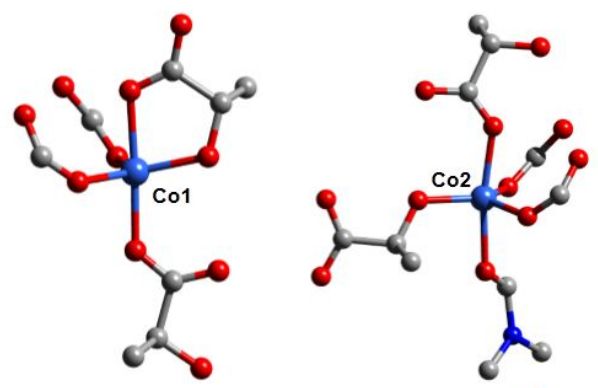

b
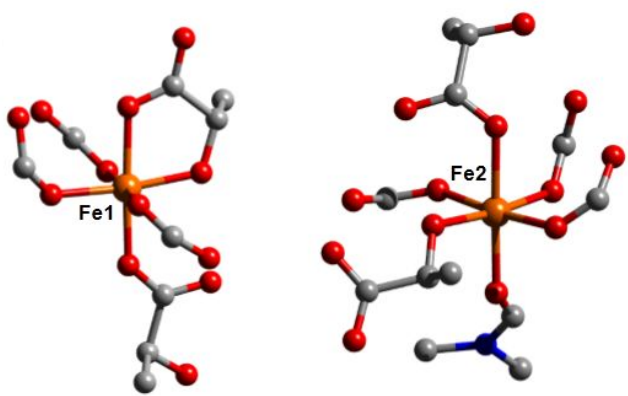

Figure S2. Coordination enviroment of metal ions in 3L (a) and 4L (b).
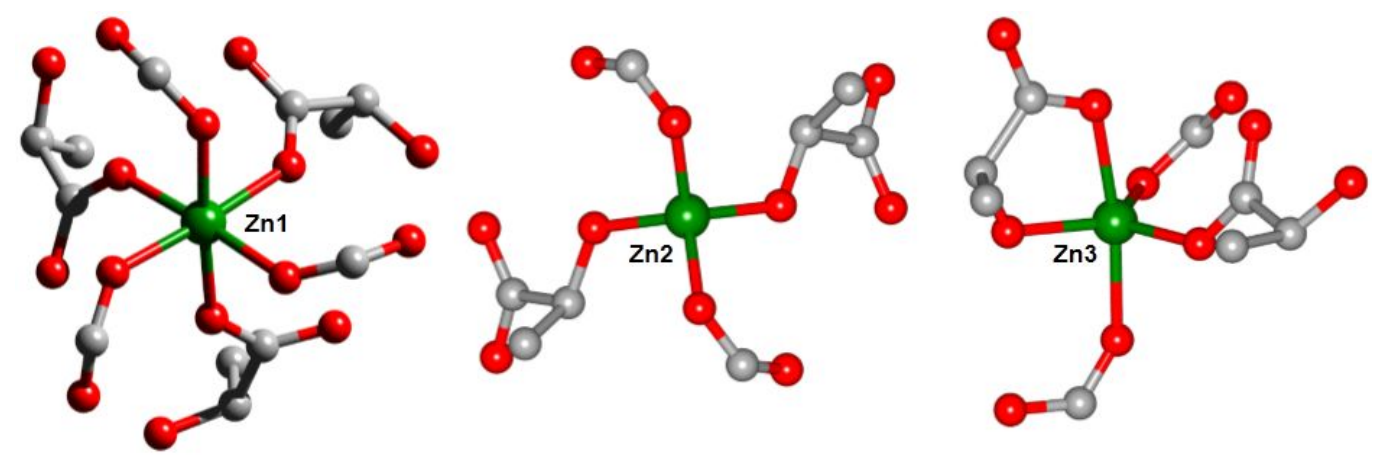

Figure S3. Coordination enviroment of metal ions in $\mathbf{5 L}$. 


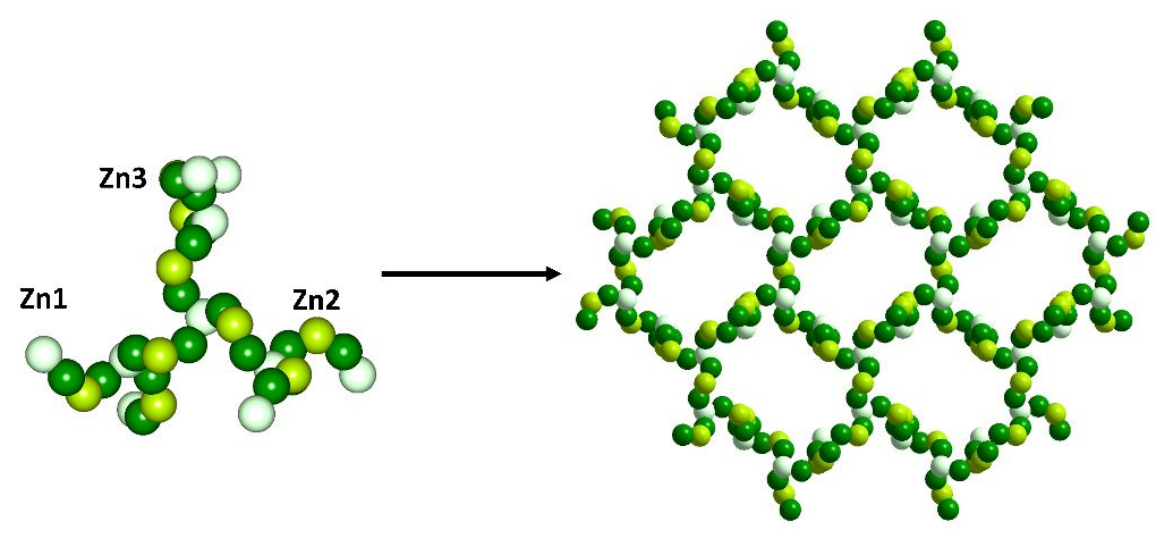

Figure S4. Arrangement pattern of metal ions in 5L.

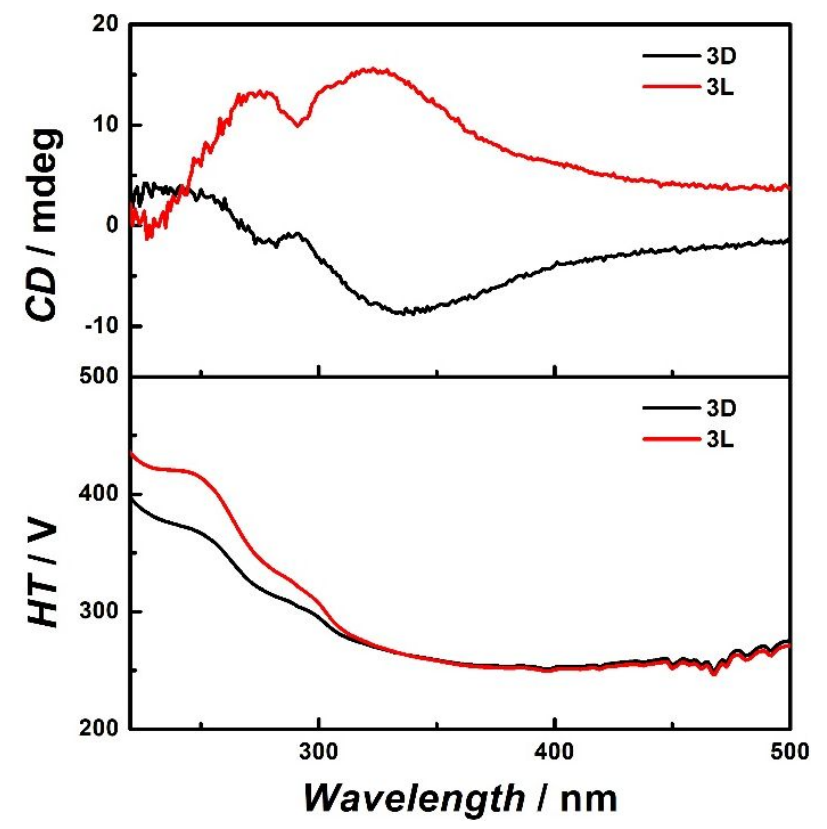

Figure S5. ECD spectra of 3D and 3L with KBr pellets. 


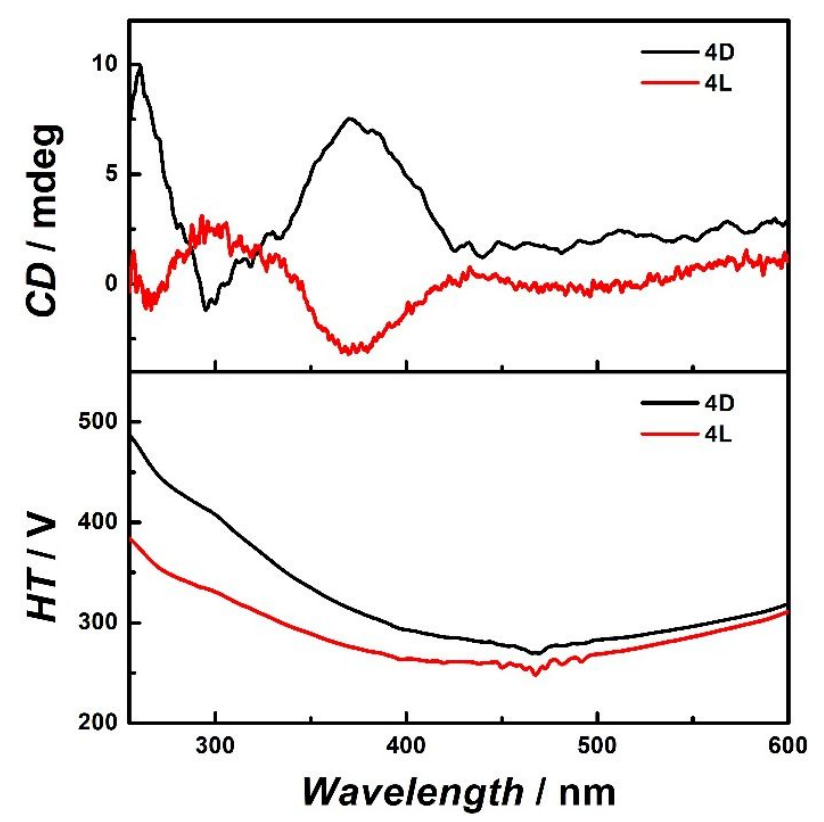

Figure S6. ECD spectra of 4D and $4 \mathrm{~L}$ with $\mathrm{KBr}$ pellets.

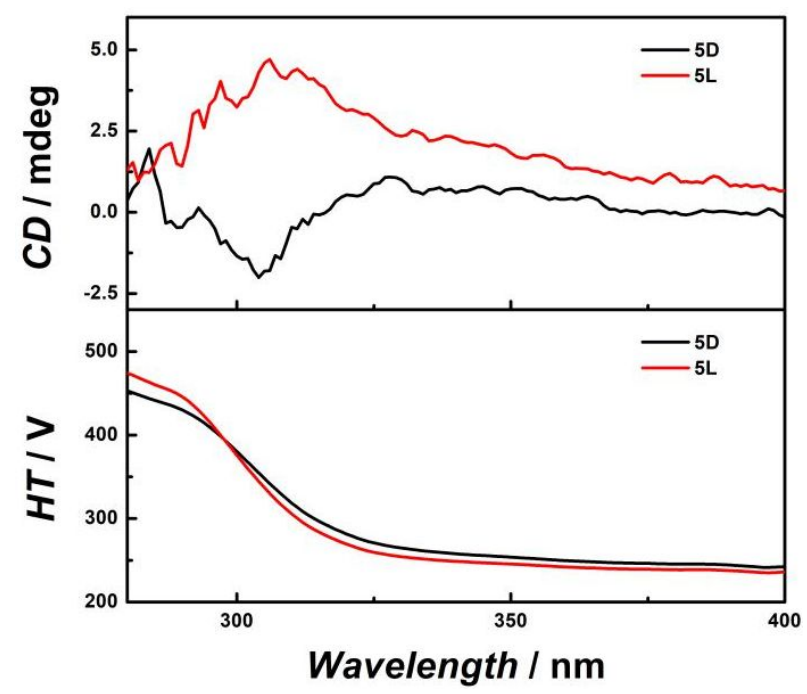

Figure S7. ECD spectra of 5D and $\mathbf{5 L}$ with $\mathrm{KBr}$ pellets. 
Table S1. Crystal parameters for 2-5.

\begin{tabular}{|c|c|c|c|c|c|c|c|c|}
\hline Compound & $2 \mathrm{~L}$ & $2 D$ & 3D & $3 \mathrm{~L}$ & 4D & $4 L$ & 5D & $5 \mathrm{~L}$ \\
\hline S. G. & $P 2{ }_{1} 2_{1} 2_{1}$ & $P 2_{1} 2_{1} 2_{1}$ & $P 2{ }_{1} 2_{1} 2_{1}$ & $P 2{ }_{1} 2_{1} 2_{1}$ & $C 2$ & $C 2$ & $P 4_{1} 32$ & $P 4_{3} 32$ \\
\hline$a / \AA$ & $\begin{array}{c}10.3111 \\
(4)\end{array}$ & $\begin{array}{c}10.2991 \\
\text { (3) }\end{array}$ & $\begin{array}{c}10.3199 \\
(4)\end{array}$ & $\begin{array}{c}10.3388 \\
(2)\end{array}$ & $\begin{array}{c}23.6044 \\
(4)\end{array}$ & $\begin{array}{c}23.5717 \\
\text { (3) }\end{array}$ & $\begin{array}{c}28.8135 \\
(3)\end{array}$ & $\begin{array}{c}28.8085 \\
\text { (2) }\end{array}$ \\
\hline$b / \AA$ & $\begin{array}{c}11.7044 \\
\text { (5) }\end{array}$ & $\begin{array}{c}11.7050 \\
(4)\end{array}$ & $\begin{array}{c}11.7682 \\
\text { (6) }\end{array}$ & $\begin{array}{c}11.7807 \\
\text { (3) }\end{array}$ & $\begin{array}{c}10.1825 \\
\text { (2) }\end{array}$ & $\begin{array}{c}10.1886 \\
\text { (1) }\end{array}$ & $\begin{array}{c}28.8135 \\
\text { (3) }\end{array}$ & $\begin{array}{c}28.8085 \\
\text { (2) }\end{array}$ \\
\hline$c / \AA$ & $\begin{array}{c}20.3910 \\
(7)\end{array}$ & $\begin{array}{c}20.3915 \\
(5)\end{array}$ & $\begin{array}{c}20.2331 \\
(10)\end{array}$ & $\begin{array}{c}20.2710 \\
(5)\end{array}$ & $\begin{array}{c}11.2450 \\
(2)\end{array}$ & $\begin{array}{c}11.2496 \\
(1)\end{array}$ & $\begin{array}{c}28.8135 \\
\text { (3) }\end{array}$ & $\begin{array}{c}28.8085 \\
\text { (2) }\end{array}$ \\
\hline$\alpha /{ }^{\circ}$ & 90 & 90 & 90 & 90 & 90 & 90 & 90 & 90 \\
\hline$\beta /{ }^{\circ}$ & 90 & 90 & 90 & 90 & $\begin{array}{c}117.352 \\
(1)\end{array}$ & $\begin{array}{c}117.352 \\
\text { (1) }\end{array}$ & 90 & 90 \\
\hline$\gamma / \circ$ & 90 & 90 & 90 & 90 & 90 & 90 & 90 & 90 \\
\hline $\mathrm{V} / \AA^{3}$ & $\begin{array}{c}2460.89 \\
(17)\end{array}$ & $\begin{array}{c}2458.21 \\
\text { (13) }\end{array}$ & $\begin{array}{c}2457.25 \\
(19)\end{array}$ & $\begin{array}{c}2468.95 \\
(11)\end{array}$ & $\begin{array}{c}2400.59 \\
(8)\end{array}$ & $\begin{array}{c}2399.68 \\
(5)\end{array}$ & $\begin{array}{c}23921.5 \\
(7)\end{array}$ & $\begin{array}{c}23909.0 \\
(5)\end{array}$ \\
\hline$D c / \mathrm{g} \cdot \mathrm{cm}^{-3}$ & 1.428 & 1.430 & 1.198 & 1.192 & 1.519 & 1.520 & 0.868 & 0.869 \\
\hline$\mu / \mathrm{mm}^{-1}$ & 1.99 & 2.00 & 10.85 & 10.80 & 10.22 & 10.23 & 1.60 & 1.60 \\
\hline Data/parameters & $5014 / 283$ & $5009 / 284$ & $3728 / 217$ & $4043 / 218$ & $4098 / 290$ & $4108 / 290$ & $6952 / 258$ & $6944 / 258$ \\
\hline $2 \theta /^{\circ}$ & $7.0-52.8$ & $7.0-52.8$ & $8.8-123.0$ & 8.6-129.6 & $8.4-133.2$ & $8.4-132.8$ & $6.8-133.2$ & 7.6-133.6 \\
\hline Obs reflns & 12072 & 12290 & 7147 & 10425 & 10143 & 10609 & 35825 & 37202 \\
\hline GOOF & 1.13 & 1.12 & 1.06 & 1.06 & 1.05 & 1.06 & 1.05 & 1.02 \\
\hline$R_{1}^{\mathrm{a}}$ & 0.051 & 0.053 & 0.076 & 0.067 & 0.067 & 0.067 & 0.052 & 0.051 \\
\hline
\end{tabular}




\begin{tabular}{|c|c|c|c|c|c|c|c|c|}
\hline$w R_{2}^{\mathrm{b}}$ & 0.126 & 0.128 & 0.220 & 0.194 & 0.192 & 0.191 & 0.152 & 0.146 \\
\hline$R$ (int) & 0.030 & 0.032 & 0.052 & 0.038 & 0.093 & 0.038 & 0.044 & 0.048 \\
\hline Flack & 0.07 (4) & $0.09(4)$ & $0.036(14)$ & $0.046(12)$ & $0.003(5)$ & $0.005(5)$ & 0.008 & $\begin{array}{r}0.012 \\
(14)\end{array}$ \\
\hline CCDC number & - & 2044330 & 2044331 & 2044332 & 2044333 & 2044334 & 2044660 & 2044619 \\
\hline${ }^{\mathrm{a}} R_{1}=\sum|| F_{\mathrm{O}}|-|$ & $\left|/ \sum\right| F_{\mathrm{o}} \mid$ & ${ }^{\mathrm{b}} w R_{2}=\left\{\sum[\right.$ & $\left(F \mathrm{o}^{2}-F \mathrm{c}^{2}\right)^{2}$ & $\sum\left[w\left(F_{\mathbf{O}^{2}}\right.\right.$ & & & & \\
\hline
\end{tabular}

Table S2. Thermodynamic data[a] ( Unit: kcal/mol ) of single left-handed ( L ) and mesotropia ( $\mathrm{DL}$ and $\mathrm{D}+\mathrm{L})^{[\mathrm{b}]}$ compounds.

\begin{tabular}{|l|l|l|l|l|l|l|l|l|l|}
\hline Comp. & $\mathbf{E}_{\mathbf{D F T}}$ & $\mathbf{S} * \mathbf{T}$ & $\left.\boldsymbol{\Delta}_{\mathbf{r}} \mathbf{S}^{* \mathbf{T}}\right)^{\mathbf{c}}$ & $\mathbf{Z P E}$ & $\mathbf{E} \mathbf{H}$ & $\mathbf{H}$ & $\left.\boldsymbol{\Delta}_{\mathbf{r}} \mathbf{H}\right)^{\mathbf{c}}$ & $\mathbf{G}$ & $\left.\mathbf{\Delta}_{\mathbf{r}} \mathbf{G}\right)^{\mathbf{c}}$ \\
\hline $\mathbf{2} \mathbf{D L}$ & -27436.48 & 130.67 & 2.48 & 705.84 & 66.13 & -26664.51 & 2.88 & -26795.19 & 0.40 \\
\hline $\mathbf{2} \mathbf{D}+\mathbf{L}$ & -27434.87 & 128.67 & 0.48 & 705.64 & 65.48 & -26663.75 & 3.65 & -26792.42 & 3.17 \\
\hline $\mathbf{2 L}$ & -27437.83 & 128.19 & 0.00 & 705.59 & 64.84 & -26667.40 & 0.00 & -26795.59 & 0.00 \\
\hline 4 DL & -36931.83 & 147.32 & 1.17 & 867.32 & 78.04 & -35986.48 & 10.12 & -36133.80 & 8.95 \\
\hline 4 D+L & -36932.90 & 146.54 & 0.39 & 868.86 & 78.29 & -35985.75 & 10.85 & -36132.29 & 10.46 \\
\hline 4L & -36942.62 & 146.15 & 0.00 & 868.02 & 78.00 & -35996.60 & 0.00 & -36142.74 & 0.00 \\
\hline
\end{tabular}

${ }^{\text {a }}$ EFFT $_{\text {D }}$ Kohn-Shame energy from DFT calculation; S*T: entropy contribution at $298 \mathrm{~K}$; ZPE: zero-point energy; E_H: thermal correction to Enthalpy; H: enthalpy; G: Gibbs free energy. Corresponding relation between them is presented as follows: $\mathrm{H}=\mathbf{E}_{\mathrm{DFT}}+\mathrm{ZPE}+\mathbf{E}_{-} \mathrm{H} ; \mathrm{G}=\mathbf{H}-\mathrm{S}^{*} \mathrm{~T}$. ${ }^{\mathrm{b}}$ DL: right-handed and left-handed carbons alternately coexist in one chain; D+L: right-handed chain and left-handed chain alternately coexist in the crystal. ${ }^{c} \Delta\left(\Delta_{r} G\right), \Delta\left(\Delta_{r} H\right)$ and $\Delta\left(\Delta_{r} S\right)$ represent the difference for Gibbs free energy, enthalpy and entropy change of the reaction respectively. 


\section{Reference}

1. Sheldrick, G. M. SHELXT - Integrated space-group and crystal-structure determination. Acta Crystallogr., Sect. A: Found. Adv. 2015, 71, 3-8.

2. Dolomanov, O. V.; Bourhis, L. J.; Gildea, R. J.; Howard, J. A. K.; Puschmann, H. OLEX2: a complete structure solution, refinement and analysis program. J. Appl. Cryst. 2009, 42, 339-341.

3. Sheldrick, G. M. Crystal structure refinement with SHELXL. Acta Cryst. 2015, C71, 3-8.

4. Spek, A. L. Single-crystal structure validation with the program PLATON. J. Appl. Crystallogr. 2003, 36, 7-13.

5. Kresse, G.; Hafner, J. Ab initio molecular dynamics for liquid metals. Phys. Rev. B 1993, 47, $558-561$.

6. Kresse, G.; Furthmüller, J. Efficiency of ab-initio total energy calculations for metals and semiconductors using a plane-wave basis set. Computational Materials Science 1996, 6, 15-50.

7. Perdew, J. P.; Burke, K.; Ernzerhof, M. Generalized gradient approximation made simple. Phys. Rev. Lett. 1997, 78, 1396-1396.

8. Blöchl, P. E. Projector augmented-wave method. Phys. Rev. B 1994, 50, 17953-17979.

9. Grimme, S.; Antony, J.; Ehrlich, S.; Krieg, H. A consistent and accurate ab initio parametrization of density functional dispersion correction (DFT-D) for the 94 elements H-Pu. $J$. Chem. Phys. 2010, 132, 154104. 
10. Paier, J.; Hirschl, R.; Marsman, M.; Kresse, G. The Perdew-Burke-Ernzerhof exchangecorrelation functional applied to the G2-1 test set using a plane-wave basis set. J. Chem. Phys. 2005, 122, 234102.

11. Paier, J.; Marsman, M.; Hummer, K.; Kresse, G.; Gerber, I. C.; Ángyán, J. G. Screened hybrid density functionals applied to solids. J. Chem. Phys. 2006, 124, 154709.

12. Paier, J.; Marsman, M.; Kresse, G. Why does the B3LYP hybrid functional fail for metals? $J$. Chem. Phys. 2007, 127, 024103.

13. Wang, V.; Xu, N.; Liu, C. J.; Tang, G.; Geng, W. T. VASPKIT: A User-friendly Interface Facilitating High-throughput Computing and Analysis Using VASP Code. arXiv:1908.08269 (https://arxiv.org/abs/1908.08269) 2019.

14. http://www.ccdc.cam.ac.uk/. CSD version 5.41, November 2019. 